\title{
Research contributions and challenges in DLT-based cryptocurrency regulation: a systematic mapping study
}

\author{
Elcelina Carvalho Silva ${ }^{1,2} \cdot$ Miguel Mira da Silva $^{2}$ (I)
}

Received: 18 April 2021 / Accepted: 9 December 2021 / Published online: 11 January 2022

(c) Institute for Development and Research in Banking Technology 2022

\begin{abstract}
The purpose of this paper is to identify research that has been carried out about cryptocurrency regulation contributions and the current challenges that need to be addressed in future studies. The methodology used to conduct this research and report the findings was systematic mapping. We use this methodology to search, identify, and select all relevant primary studies on cryptocurrency regulation. The findings reveal that the key cryptocurrency regulation research topics are distributed governance, central bank digital currency, monetary policy, cryptocurrency adoption, security, regulation, cryptocurrency market, cybercrime economy and money laundering. The research proposals for cryptocurrency regulation comprise tools, protocols, methods, models, frameworks and, knowledge. The cryptocurrency regulatory challenges are cryptocurrency adoption, central bank digital currency regulation, accounting for cryptocurrencies and risk for cryptocurrencies. This systematic mapping provides an overview of the solutions proposed to regulate cryptocurrency as well as the current research challenges.
\end{abstract}

Keywords Cryptocurrency $\cdot$ Regulation $\cdot$ Contributions $\cdot$ Challenges $\cdot$ Systematic mapping

\section{Introduction}

Blockchain technology is defined as a distributed database of records or public ledger of all transactions or digital events that have been executed and shared among participating parties where each transaction in the public ledger is verified by consensus of a majority of the participants in the system, and once entered, the information can never be erased [1].

Cryptocurrency is a peer-to-peer version of electronic cash, implemented though digital signatures, that allows online payments to be sent directly from one party to another without a financial institution [2]. Today, cryptocurrencies are largely accepted because they allow members of society who could not open a regular bank account to participate in financial transactions via an internet-based altcoin account operable from any smartphone [3].

Elcelina Carvalho Silva

elcelina.silva@docente.unicv.edu.cv

Miguel Mira da Silva

mms@tecnico.ulisboa.pt

1 Faculty of Science and Technology, University of Cape Verde, Palmarejo Grande, CP 379C Praia, Cabo Verde

2 Instituto Superior Técnico, University of Lisbon, Av. Rovisco Pais, 1049-001 Lisboa, Portugal
From this perspective, blockchain may be comparable to the internet of the 1990s [4] and the cryptocurrency market repeats the history of the 1990s stock market when innovative technologies such as electronic trading platforms began to emerge [5].

Cryptocurrency is defined as a type of unregulated digital money which is issued and usually controlled by its developers, and used and accepted among the members of a specific virtual community [6]. According to CoinMarketCap website, ${ }^{1}$ accessed in February 2021, nowadays there exist more than 3,207 different types of cryptocurrencies with market capitalization. Bitcoin and Ethereum are at the top of the market capitalization list.

The growth of cryptocurrency brings with it associated risks such as black markets, technology hacking, speculation bubbles, highly power-intensive energy consumption [7], and credit, operational and liquidity risks without legal protection [6]. The use of cryptocurrency as a payment alternative for those engaged in drug trafficking and money laundering, results in calls from legislators and law enforcement agencies to impose strict regulation on the use of virtual currency [8].

The possibility of using cryptocurrency without the intervention of financial institutions that can be targeted

\footnotetext{
${ }^{1}$ https://coinmarketcap.com/
} 
by governments, is motivating corrupt firms and criminal organizations to convert their earnings into cryptocurrency and then transfer these funds anywhere in the world to evade tax authorities [9].

Moreover, various positions have been taken by the media regarding cryptocurrency regulation, such as news regarding the legal status of cryptocurrency, calls for banning cryptocurrency and its use in financial transactions, news related to its possible treatment under securities market law, and news reporting the introduction of central bank digital currency [10].

On one hand, we have private cryptocurrencies such as Bitcoin and Ethereum that are cryptographically protected, decentralized digital currencies used as a means of exchange and not controlled by any single entity but maintained by their anonymous users connected via peer-to-peer networks $[6,11]$. On the other hand, several countries are searching for ways to introduce cryptocurrency regulation through Central Bank Digital Currency (CBDC) [12].

The goal of this systematic mapping is to identify the research contributions and the current research challenges on cryptocurrency regulation. The methodology used to conduct this research is found in the guidelines for conducting systematic mapping studies in software engineering [13].

The structure of this review is organized into seven sections: here in Sect. 1, we have introduced this research paper with the objective, methodology and structure of this systematic mapping. In Sect. 2, the theoretical background of blockchain-based cryptocurrency regulation is discussed. In Sect. 3, the related works in this domain are presented. The methodology adopted to create this mapping is detailed in Sect. 4. The results are presented in Sect. 5 and discussed in Sect. 6. The limitations that may threaten the validity of the results are identified in Sect. 7. Finally, concluding remarks are made in Sect. 8.

\section{Theoretical background and motivation}

Cryptocurrency is revolutionizing many traditional banking services with better transaction security and faster exchanges of money [14]. It has the potential to become a disruptive innovation because it will define a new paradigm of decentralization of trust in secure electronic transactions without the need for a central control authority [15].

The crypto encyclopedia defines cryptocurrency as digital currency in which encryption techniques are used to control the generation of units of currency and verify the transfer of funds, operating independently of one single central unit. It is a distributed ledger technology (DLT), a technology based on the principle of distributed ledgers, that is a digital system recording and storing data and which is consensually shared and synchronized across a geographically spread network across multiple sites, institutions and/or geographies [16].

Currency transactions between companies or persons are generally centralized in servers that are controlled by third party organizations while cryptocurrency is stored in a decentralized network where there is no control of the third party over data and transactions. There are various Distributed Ledger Technologies such as blockchain, sidechain, tangle and hashgraph where the implementation of these differ substantially based on their data structures, consensus protocol and fault tolerance, among others [17].

Cryptocurrency businesses oftentimes raise money through an initial coin offering (ICO) that is an unregulated means of crowdfunding applied by cryptocurrency businesses as an alternative to the rigorous and regulated capital-raising process required by venture capitalists, banks, or stock exchanges. In an ICO, a percentage of the newly issued cryptocurrency is sold to investors in exchange for legal tender or other cryptocurrencies such as Bitcoin [16].

The decentralized system of cryptocurrencies has made global monetary systems more dynamic and thus more prone to misuse, and as well has posed a threat to financial stability [7]. Moreover, uncontrolled use of this technology threatens to accelerate socio-economic problems, especially money laundering, fraud, cybercrime, and market instability [18].

Cryptocurrency markets have been largely unregulated which has led to volatility in the market, and inevitably, to reports of significant fraud, theft, and price manipulation [19]. Their adoption and transaction volumes could threaten the overall market and price stability, thus posing risks to markets and their regulators [8].

There are also regulatory issues with the use of smart contracts, and the management and handling of litigation and disputes can be problematic. Furthermore, the irreversibility in the smart contract system is particularly risky [20]. Government regulation is necessary to deter price manipulation and fraud, but the lack of regulation is why many investors buy cryptocurrencies [19].

Central banks need to define the nature and outlook for digitalization of money, to safeguard payment business [21]. For policies to remain effective, and especially in case the market further develops and international arbitrage increases, rules and enforcement will need to be coordinated and enforced across the globe [22].

Cryptocurrency poses equally specific challenges to the financial and monetary systems, such as the potential threats to price stability, to the smooth operation of payment systems, to the implementation of monetary policy, to the prudential supervision of credit institutions, to the stability of the financial system and to the central banks' exclusive right to issue base money [23]. 
The rapid growth of cryptocurrencies presents a challenge to governments around the world given that the wide acceptance of cryptocurrencies has the potential to disrupt regulated payment systems and affect the implementation of monetary policy [7]. Authorities will need to vigilantly monitor developments and address regulatory issues arising from the global dimension of cryptocurrencies [22].

Cryptocurrency impact on central banking has sparked a policy debate on how to address the potential threats of cryptocurrencies [23]. Central banks have expressed various positions with respect to cryptocurrency including central banks' policy to lead further development of cryptocurrency by restrictions, robust surveillance and licensing [21].

The Bank International of Settlements, in January of 2020 , published a survey applied to $80 \%$ of central banks in the world, revealing that central banks assumed that their objectives in the central bank digital currency (CBDC) research are financial stability, monetary policy implementation, financial inclusion, domestic and cross-border payment efficiency, and safety/robustness of the payment systems. Some central banks reported other motivations such as reducing costs, improving know-your-customer, counteringthe-financing-of-terrorism ("KYC/CFT") and public access to central bank money [24].

The results of a survey carried out among more than 60 central banks in late 2020 [25] about their engagement in CBDC work, their motivations and their intentions regarding CBDC issuance, show that the vast majority of central banks in the survey-86\% - are exploring CBDCs, and their work continues apace amid the Covid-19 pandemic. As a whole, central banks are moving into more advanced stages of CBDC engagement, progressing from conceptual research to practical experimentation.

Decentralized Finance (DeFi) is also being discussed by various stakeholders of financial market, for example, Bank of America which sees DeFi 'Potentially More Disruptive Than Bitcoin' according CoinDesk [26] referencing "Commodity Strategist-Bitcoin's Dirty Little Secret" published by Bank of America in 17 March 2021 [27].

DeFi uses cryptocurrency and blockchain technology to manage financial transactions, with aims to democratize finance by replacing legacy, centralized institutions with peer-to-peer relationships that can provide a full spectrum of financial services, from everyday banking, loans and mortgages, to complicated contractual relationships and asset trading by disempowering middlemen and gatekeepers, and empowering everyday people via peer-to-peer exchanges [28].

Citibank's Global Perspective and Solutions (Citi GPS) entitled Future of Money: Crypto, CBDCs and 21st Century Cash, points its searchlight on MakerDAO (MKR) and the importance of decentralized finance (DeFi) [29] as a decentralized organization dedicated to bringing stability to the cryptocurrency economy using Maker Protocol which employs a two-token system [30].

Considering the social, financial, technological, and legal impact of cryptocurrency usage that is motivating changes in regulation, topics such as money laundering $[8,9]$, financial stability $[11,31]$ and financial technology (FinTech) [5, 23] are important drivers to regulate cryptocurrency. FinTech products have opened the door to many new opportunities for consumers, investors, and businesses, but with these opportunities come new challenges for regulators and policymakers to face key choices as they adapt to meet the needs of this constantly changing landscape while keeping investors and consumers safe [32].

Research on how FinTech is contributing to financial innovation in the market, such as the creation of new financial asset classes and derivatives that are facilitated by technology is being researched by Mitra and Karathanasopoulos [33]. They emphasize that financial technology could play a significant role in new risk management approaches highlighting that in terms of implications for policy and management within the banking sector (especially with respect to IT managers), the firm should take into account the relative value and the risk management contributions that operations potentially provide.

Several authors in their research articles call for antimoney laundering and for combating the financing of terrorism (AML/CFT) regulation [8, 22, 34, 35], central bank digital currency (CBDC) $[20,21,23]$ and Decentralized Finance regulation [36]. Others authors call for specific regulation and guidelines such as initial coin offerings (ICOs) [4, 37], guidance for accounting for cryptocurrencies and IFRS [38], regulation of virtual currency for taxation purposes [6], and blockchain General Data Protection Regulation [37].

The Bank for International Settlements (BIS) created the BIS Innovation Hub foster innovation and greater collaboration amongst the global central banking community. This initiative includes central bank digital currency (CBDC) research projects connected with other projects related to payment system such as financial market infrastructures (FMIs), open finance, regtech and suptech solutions, and cyber security [39].

The European Central Bank (ECB) has digital euro in the investigation phase [40], and the Central Banks of several countries are researching retail and wholesale CBDCs including the Central Banks of Brazil, the Bahamas, Canada, China, India, Japan, the Russian Federation, South Africa and others according the last working paper entitled "Rise of the central bank digital currencies: drivers, approaches and technologies" published by Bank for International Settlements (BIS) [41].

In this context, China, Britain, Japan, the United States, Germany and Italy are blocking operations of the giant decentralized crypto exchange, Binance, because it is 
unlicensed to operate [42]. In response, Binance announced on its website that they will be winding down support for stock tokens on Binance.com to shift their commercial focus to other product offerings and that stock tokens are unavailable for purchase [43].

Philipp Sandner [44], defines DeFi as an umbrella term encompassing the vision of a financial system that functions without any intermediaries, such as banks, insurers or clearinghouses, and is operated just by the power of smart contracts [44]. This researcher and professor at Frankfurt School Blockchain Center argues that financial innovations can be freely developed and implemented in DeFi without regard to regulatory boundaries but, the absence of common legislative and political principles certainly has major disadvantages, thereby underlining the fact that everything in DeFi is based on smart contracts, and hence vulnerabilities in the code of smart contracts is a fundamental risk for DeFi users.

Tony McLaughlin is his article entitled “A Treasurer's Guide to the Future of Money" [45] says that the commercial banking system and FinTechs could be caught in the crossfire of a battle between CDBCs and privately issued stable coins for monetary supremacy. This competition for deposits and payment services from two entirely new fronts could seriously impact financial stability of the banking system and the process of credit creation. He concludes that if the nature of money changes, so will the role of the Treasurerthis is true whether the future belongs to CBDC, cryptocurrencies or a fiat currency system that is hyper-connected and always on.

Various players are looking for cryptocurrency such as central banks, regulated financial institutions, telecommunications agencies, researchers, FinTech and other types of decentralized autonomous organizations promoting hot discussion of regulation from different perspectives such as financial, law and technology regulation, but is not clear if the regulation will be implemented only through CBDC or if regulations would also include, FinTechs and BigTechs within its scope.

The mechanisms for using innovative financial technologies need to be refined and the regulatory experience of different countries needs to be integrated [5]. The perspective of Azal [7] is that the technology has always preceded regulation, making regulatory options more complicated, demanding regulators meet this technological challenge and take a proactive approach to harnessing this complex modern technology.

Given the impact of cryptocurrency usage on the financial system, the new innovative start-ups using cryptocurrency and the increase in cybercrimes associated with cryptocurrency, we observed an absence of literature in cryptocurrency regulation to help understand what is being done to regulate cryptocurrency.
Kichenham et al., argue that in systematic mapping the search process is defined by topic area, while in systematic literature review the process is defined by the research question [46]. Systematic mapping studies are used to structure a research area, while systematic reviews are focused on gathering and synthesizing evidence [13]. The research questions in systematic mapping are general as they aim to discover research trends, while in systematic literature review the research question is specifically related to outcomes of empirical studies [46].

In our research, we did not find any systematic review or any systematic mapping on cryptocurrency regulation. Two studies related to cryptocurrency in the financial sector were found, but neither of these studies focused solely on cryptocurrency regulation.

The first study is a systematic literature review of “Towards Central Bank Digital Currency" [47] supplemented by academic and central bank publications to assess both the current state of academic research on CBDCs as well as research by major central banks. This study combined the various research fragments that currently exist to provide a comprehensive overview on the cryptocurrency regulation topic focusing on CBDC. As a contribution, several gaps in the scientific literature were identified, such as design properties, technical implementations as well as legal and societal aspects that have not been researched thoroughly.

The second study is a systematic mapping study of blockchain applications for Central Banks [48], focused on scientific papers, to analyse and map the gaps in scientific literature by exploring trends in peer-reviewed research contributions through thematic categorization of academic literature on Distributed Ledger Technology (DLT) use-cases for services, and operations and functions performed by central banks. As result, the study found that the most researchintensive use-cases are those for: (1) Central Bank Digital Currency (CBDC); (2) Regulatory Compliance; (3) Payment Clearing and Settlement Systems (PCS) operatedby central banks; (4) Assets Transfer/Ownership; and (5) Audit Trail.

We observed that these studies focused on central banks' cryptocurrency regulation and not on cryptocurrency regulation in the general sense, thus indicating a significant gap in the research on cryptocurrency regulation: the first paper highlights the call for central bank regulation of cryptocurrency and regulatory compliance [48], while the second paper emphasizes gaps in CBDC scientific literature such as legal and societal aspects that have not been researched thoroughly [47].

We also did not find any research study that maps the current research approach in cryptocurrency regulation that provides researchers, practitioners and regulators an overview that could help them take the research on cryptocurrency regulation even further. 
Fig. 1 Systematic mapping process [13]

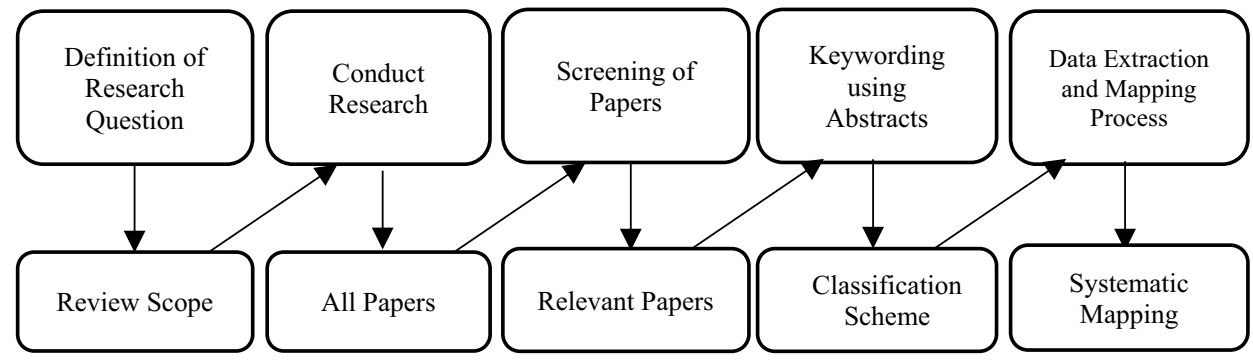

In this context, we understand that mapping the research contributions and challenges in DLT-Based Cryptocurrency regulation could help senior researchers, $\mathrm{PhD}$ Students, central banks, regulated financial institutions such as banks and insurance company, non-regulated institutions such as FinTechs to understand the state of the art of cryptocurrency regulation.

The nonexistence of research on cryptocurrency regulation that identifies, categorizes and analyses the literature and research gaps in this particular research area, especially information of evidence for future research direction, motivated us to conduct this systematic mapping focusing on the understanding of the current research that are being done by researchers and practitioners to better conduct the future research in cryptocurrency regulation solutions.

The systematic mapping allows us to find the various perspectives of cryptocurrency regulation and details of research contributions such as knowledge, framework, method, model, protocol, and tools created to resolve problems of cryptocurrency regulation.

The goal of this systematic mapping is to identify the existing research regarding cryptocurrency regulation and the challenges that need to be addressed in future studies. This goal leads us to define the following research questions (RQs):

RQ1: what are the publication approaches used for cryptocurrency regulation? This question is motivated by the need to understand the research maturity and we did this by analysing the research methods and types of the selected studies.

RQ2: what are the current research topics on cryptocurrency regulation? Mapping the current research topics on cryptocurrency regulation will help other researchers and practitioners gain a better understanding of the current research topics and take the research on cryptocurrency regulation even further.

RQ3: what are the research contributions to cryptocurrency regulation? This question evaluates the current research contributions to cryptocurrency regulation to understand what is being done to resolve the various problems associated with unregulated cryptocurrency.
RQ4: what are the research challenges for cryptocurrency regulation? The list of the current research challenges in cryptocurrency regulation could help other researchers and practitioners focus their research on the areas that require more attention.

\section{Research method}

This study has been conducted as a Systematic Mapping, a methodology used to structure a research area and to identify existing systematic maps concerning topics investigated, frequency of publication over time, and venues of publication [13].

We chose the systematic mapping process as our research methodology because the systematic map is a method that is effectively used in software engineering to build a classification scheme and structure research field of interest [49].

Figure 1 presents the mapping process including the definition of the research question, search, study selection, data extraction, data analysis and classification steps.

We followed the systematic mapping process proposed by [13]. We also used the guidelines for systematic literature review [50] to search for relevant papers. In complement, we used the PRISMA checklist [51] to materialize the proposed methodology in order to conduct the systematic mapping [13].

The research was conducted between June and July of 2020. We updated it in February 2021. The results of this mapping study helped us to identify and map research areas related to cryptocurrency regulation and current research challenges.

In the following section, we present the search protocol that was developed by the second author and reviewed by the first author.

\subsection{Search strategy}

Based on the research goal of this study, we defined the Population, Intervention, Comparison, Outcomes and Context (PICOC) [50] using the following terms:

- Population: Central Bank, researcher. 
- Intervention: Regulation, legislation, law, standards.

- Comparison: Cryptocurrency.

- Outcome: Financial system, financial stability, systemic risks, security risks.

- Context: Regulators, regulated.

Based on the combination of PICOC terms defined, we used the Boolean "OR" to join the terms of each part and then we generated the search string.

\subsection{Study selection}

We excluded articles based on titles and abstracts, as well as full-text reading and quality assessment. The study selection is used to identify those primary studies that provide direct evidence for the research questions [50]. The content of selected primary studies should report the phenomenon of cryptocurrency regulation, the needs and proposed solutions for cryptocurrency regulation, the vision of regulators such as central banks, the research on cryptocurrency regulation on financial systems for the regulators, the regulated and other groups of interest, or innovation initiatives on regulation, legislation, law, and standards for cryptocurrency.

From this focus, we developed the following inclusion criteria:

1. Scientific: short or full paper focused on cryptocurrency regulation;

2. Published in peer-reviewed journals, conference proceedings, open-access journals;

3. Empirical research (qualitative and quantitative).

We excluded papers with the following features:

1. Not written in English;

2. Published before 2008 (the first cryptocurrency-Bitcoin-was created in 2008 by Satoshi Nakamoto);

3. Duplicated articles;

4. Not fully accessible;

5. Extended abstracts or "works-in-progress";

6. Not scientific (report, book);

7. Regarding a topic unrelated to cryptocurrency regulation.

The inclusion criteria ensure that select papers focused on the context of this systematic mapping with high-quality work reporting original research. The exclusion criteria exclude papers not written in English and before 2008, incomplete versions of studies, papers focusing only on decentralized ledger technology, blockchain technology or its application, and papers about cryptocurrencies such as Bitcoin, Ethereum, and Monetha among others.

\subsection{Data extraction}

The extraction was done before a full reading of selected articles. To answer the defined research questions, we developed a data extraction sheet in accordance with [49, 52] and organized the data extraction results into two categories: topic-independent classification and topic-specific classification.

In the topic-independent classification, we extracted research method and research type.

The classification of empirical research methods has also been frequently reported in mapping studies to describe the research methodologies that are frequently applied in software engineering such as survey, case study, controlled experiment, action research, ethnography, simulation, prototyping, and mathematical analysis [52]. The authors showed that these research methods could belong to various types, giving examples of how some experiments with students could be classified as validation research, while some experiments with practitioners could be classified as evaluation research.

Empirical research seeks to explore, describe, predict, and explain natural, social, or cognitive phenomena by using evidence-based observation or experience, by obtaining and interpreting evidence by experimentation, systematic observation, interviews or surveys, or by the careful examination of documents or artefacts [53]. These authors categorize empirical research studies as follows:

- Survey: investigates relationships and outcomes, studying a large number of variables using a large sample size and rigorous statistical analysis.

- Case study: investigates a contemporary phenomenon within its real-life context.

- Experiment: investigates causal relations and processes, conducted when the investigator wants control over the situation, with direct, precise, and systematic manipulation of the behaviour of the phenomenon to be studied.

- Action research: focuses particularly on combining theory and practice, contributing to the acquisition of new theoretical knowledge, with an iterative process involving researchers and practitioners acting together on a particular cycle of activities, including problem diagnosis, intervention, and reflective learning.

For the research type, we used the requirements engineering (RE) paper classification and evaluation criteria categorization of research type according to [54]:

- Evaluation research: this is the investigation of a problem in RE practice or implementation of a RE technique in practice. Causal properties are studied empirically, such as case study, field study, field experiment, survey, etc. 
Table 1 Data extraction form

\begin{tabular}{|c|c|c|c|}
\hline Data item & Value & & \\
\hline Year of Publication & Integer & \multirow{4}{*}{$\begin{array}{l}\text { Topic-inde- } \\
\text { pendent clas- } \\
\text { sification }\end{array}$} & \multirow[t]{4}{*}{ RQ1 } \\
\hline Source & String & & \\
\hline Empirical research & Surveys; case study; experiment; action research & & \\
\hline Research type & $\begin{array}{l}\text { Evaluation; solution; validation; philosophical; opin- } \\
\text { ion; experience }\end{array}$ & & \\
\hline Research topics & String & \multirow{3}{*}{$\begin{array}{r}\text { Topic-specific } \\
\text { classification }\end{array}$} & RQ2 \\
\hline Research contribution & String & & RQ3 \\
\hline Research challenges & String & & RQ4 \\
\hline
\end{tabular}

- Proposal of the solution: this paper proposes a solution technique and argues for its relevance, without a fullblown validation.

- Validation research: this paper investigates the properties of a solution proposal that has not yet been implemented in RE practice. The solution may have been proposed elsewhere, by the author or by someone else. Possible research methods are experiments, simulation, prototyping, mathematical analysis, mathematical proof of properties, etc.

- Philosophical papers: these papers sketch a new way of looking at things, a new conceptual framework, etc.

- Opinion papers: these papers contain the author's opinion about what is wrong or good about something, how we should do something, etc.

- Personal experience papers: in these papers, the emphasis is on what and not on why. The experience may concern one project or more, but it must be the author's personal experience.

In the topic-specific classification, we used the guideline [13] to map the research topics, contributions and challenges that emerged in the selected papers. First, we read abstracts and we looked for the contribution type of the paper. When the abstracts were too poor in quality to ensure clarity of the contribution to be chosen, we decided to study the introduction and the conclusion sections of the paper.

For the research challenges, we read the conclusion and we identified the gaps in the current research as pointed out by authors. We considered these gaps as a set of research challenges that need to be solved in future research on cryptocurrency regulation. When the conclusion is not clear or with poor information, we chose to study the abstracts and introduction to identify the research gaps.

Then, we combined a set of keywords from different papers to develop a high-level understanding of the nature of the research challenges, grouping papers with the same focus. After having identified the main categories based on a set of keywords chosen, we created the list of keywords that defined each category and we mapped the list of research challenges.
We designed the data extraction form (presented in Table 1) to extract and manage the data extracted from each selected paper.

The extraction was done after the full reading of selected articles. The extraction was performed by the second author and reviewed by the first author by tracing back the information in the extraction form to the statements in each paper and checking their correctness.

\section{Results}

In this section, we report the results of the research questions via this systematic mapping. Figure 2 shows the number of mapping studies identified within the years 2008-2020. The mapping methodology was applied, creating a data extraction template on Parsifal, an open-source web application to perform systematic mapping in software engineering. Then we used Excel to make the data analysis.

We imported 368 documents from the ACM $(n=75)$, IEEE $(n=77)$, Google Scholar $(n=34)$, ScienceDirect (78), Scopus $(n=70)$, and Springer $(n=29)$. Additional papers were identified with a manual search of the reference lists of key studies and more papers were included $(n=5)$.

The selection process is reported as recommended by the PRISMA guideline [51]. We removed 29 duplicates, leaving 339 papers. By screening the title and the type of document, we removed 11 documents that were not scientific papers including extended abstract or poster, work in progress, books, and book chapters. Based on the title and abstract, 234 papers were removed because the topic did not relate to cryptocurrency regulation, leaving 105 papers.

Based on defined exclusion criteria, we excluded 63 articles papers not related to the goal, papers related to cryptocurrency regulation on specific country or region, leaving 42 papers. Based on quality assessment, 10 papers were removed based on cut-off score, leaving 32 papers for a full reading. 
Fig. 2 Selection process, based on the PRISMA guideline [51]

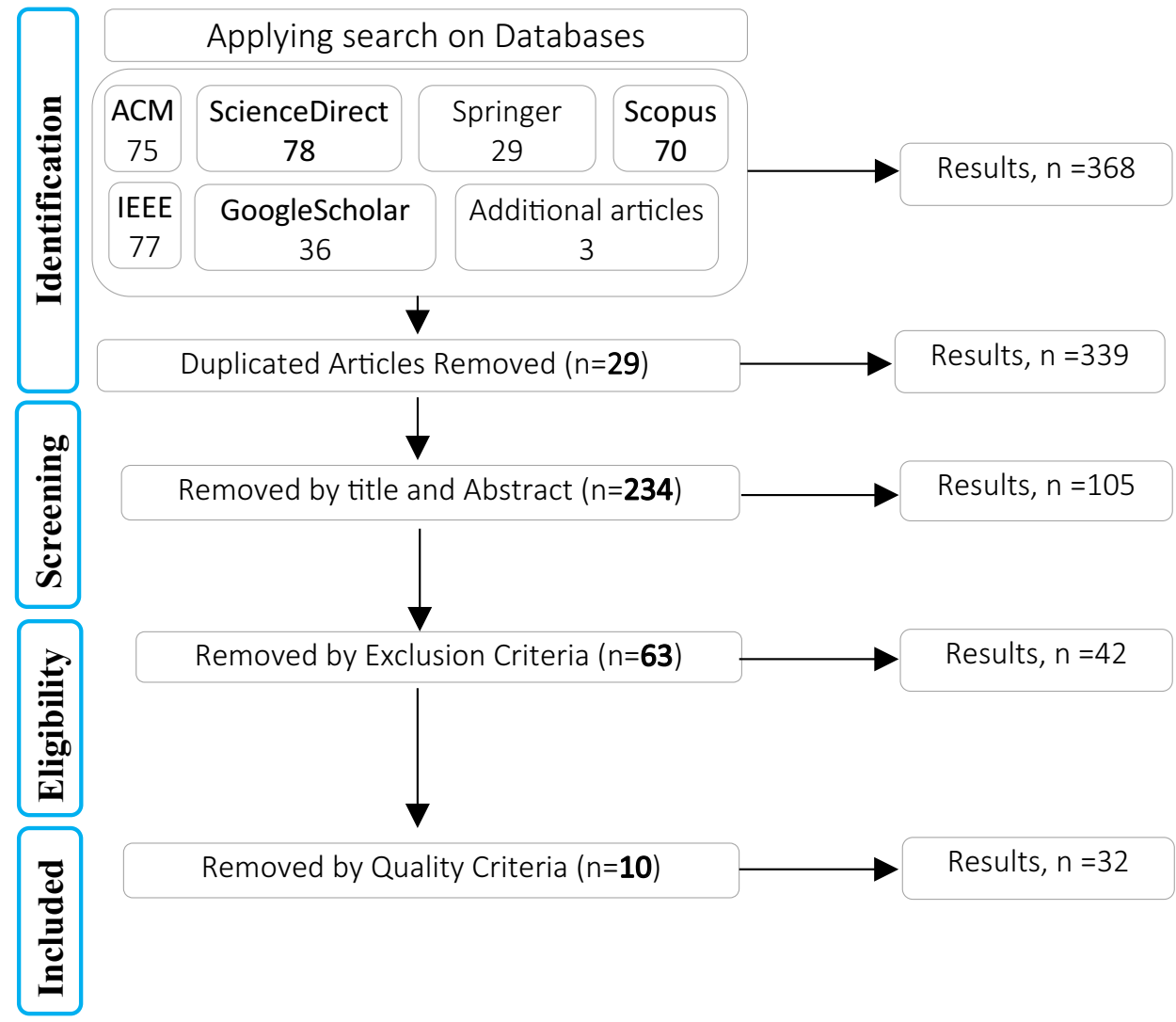

10

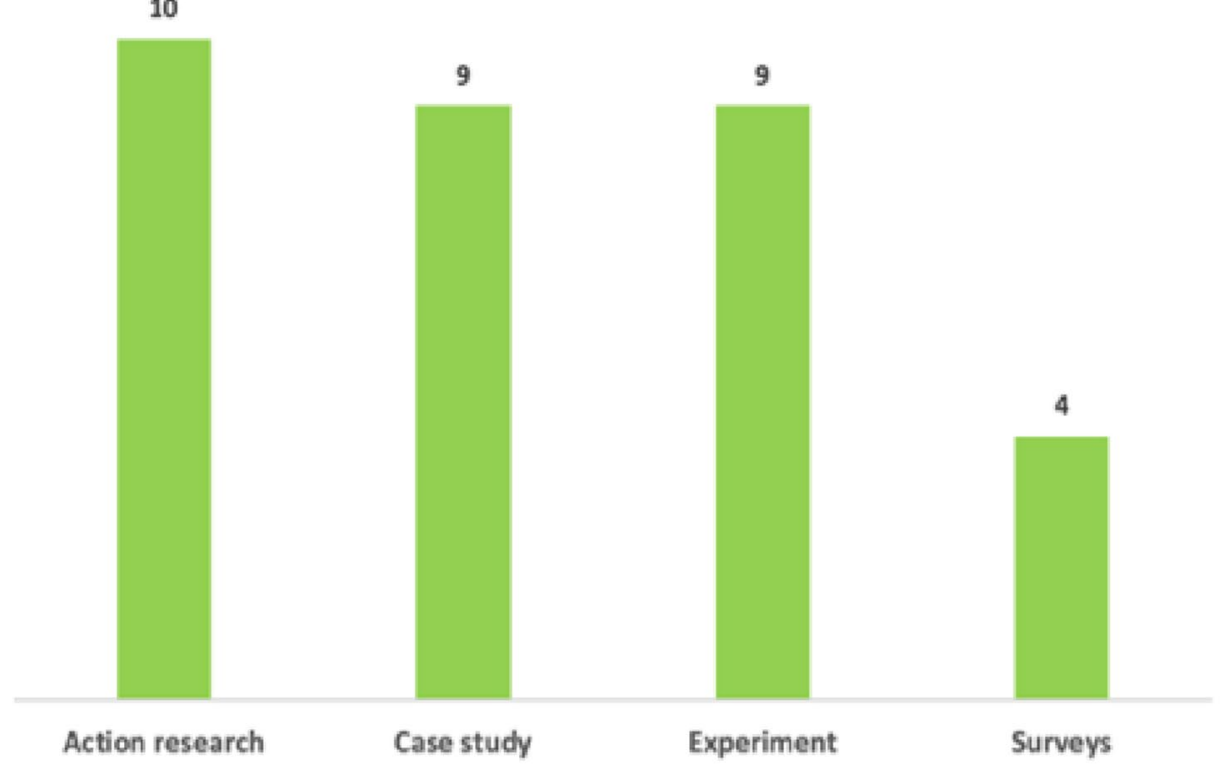

\subsection{RQ1: what are the publication approaches used for cryptocurrency regulation?}

In this section, we present how research on cryptocurrency regulation is being done by the scientific community by mapping the study method and the type of research of the selected studies.

In this research we classified the research method according to a classification model [53] and the research type based on classification and evaluation criteria [54]. The results are presented in Figs. 3 and 4. 
Fig. 4 Research type

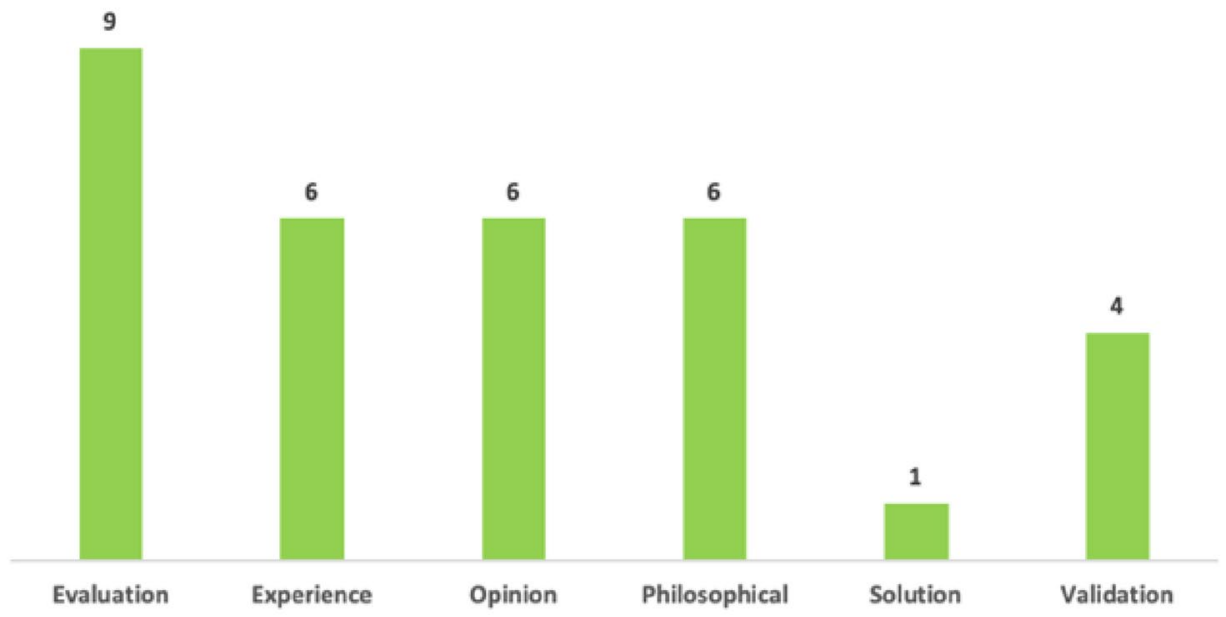

The action research method represents $31.3 \%$ of selected papers, indicating that some research on cryptocurrency regulation is being done through a combination of theory and practice.

Experiment and case study papers counted 9 papers each, and each representing $28.1 \%$ of the selected papers. The result for case studies suggests that research on cryptocurrency, as contemporary phenomenon within the regulatory context, is increasing. Given the results for experiments, we observed that experiments are being conducted on regulatory processes to control the associated risks of unregulated cryptocurrency via direct, precise, and systematic manipulation of the behaviour of the cryptocurrency.

Among research types, evaluation represents $28.1 \%$ of the selected papers. This result reveals that the research papers were often focused on the problems of cryptocurrency and implementation of proposals for cryptocurrency regulation.

The papers that contain the author's opinion about problems related to unregulated cryptocurrency and how regulation should resolve these problems, represented $21.9 \%$ of selected papers. The philosophical papers that sketched a new approach or vision about cryptocurrency regulation represents $18.8 \%$ of selected papers.

Papers that emphasised the author's personal experience on projects related to the solution of cryptocurrency regulation represent $15.9 \%$ of selected papers. The validation type counted 4 papers, representing $12.5 \%$ of papers, focused on the investigation of a solution proposed for cryptocurrency regulation but that has not yet been implemented in practice. The solution type had only one paper revealing the need for more research of this type.

This section indicates the level of maturity of research into cryptocurrency regulation by analysing the research method and type of the selected studies. This information could help improve the understanding of how the research on cryptocurrency regulation is being done.

\subsection{RQ2: what are the current research topics on cryptocurrency regulation?}

This section provides an overview of the current research topics on cryptocurrency regulation, using the keywording technique. We classified all the relevant papers by using the keywording technique explained by [13].

Table 2 shows the classification of the research topics of the selected papers.

\subsubsection{Distributed governance}

This category included studies of challenges posed by distributed governance such as the balance of integrity and autonomy, decision-rights, control mechanisms, accounting for cryptocurrencies acquired for investment purposes, the management of cryptocurrency through distributed networks, solutions to reduce the aggregated cost of knowyour-customer (KYC) requirements, and cryptocurrency compatibility with data protection.

Table 2 Research topics

\begin{tabular}{ll}
\hline Research topics & Papers \\
\hline Distributed Governance & {$[35,37,38,56,63]$} \\
Central Bank Digital Currency & {$[21,23,47,62]$} \\
Regulation & {$[6,60,65,66]$} \\
Cybercrime Economy & {$[34,57,59,61]$} \\
Security & {$[4,18,58]$} \\
Cryptocurrency Adoption & {$[3,15,19]$} \\
Cryptocurrency Market & {$[7,11,22]$} \\
Monetary Policy & {$[5,20,31,64]$} \\
Money Laundering & {$[8,9]$} \\
\hline
\end{tabular}




\subsubsection{Central bank digital currency}

This category included studies of research and evaluation of central banks' digital currency, central banks' desire to lead further development of cryptocurrency, cryptocurrency design features such as value-based, account-based, wholesale, retail-oriented, interest-bearing, and non-interest bearing.

\subsubsection{Monetary policy}

This category comprised studies of the impact of cryptocurrency on monetary policy, the monetary policy risks of digital currencies, the analysis and estimation of risks related to unregulated virtual currencies to monetary policy.

\subsubsection{Cryptocurrency adoption}

Articles in this category included studies of cryptocurrencies from a business ethics perspective, the categorization and identification of factors that could influence the intention to use cryptocurrency, the disruptive innovation of cryptocurrencies in consumer acceptance, and cryptocurrency acceptance models for $\mathrm{C} 2 \mathrm{C}$ e-commerce.

\subsubsection{Security}

This category included studies of security issues of blockchain-based transactions in 5G networks, policy specification and verification of transactions based on smart contracts in next-generation mobile networks, transaction protocols, and platforms for micropayments and initial coin offerings (ICOs).

\subsubsection{Regulation}

This category covered studies of evaluation of the legal framework of cryptocurrency, intersection and interactions between conventional law produced and enforced by national legal systems (i.e., the 'code of law') and the internal rules of blockchain systems (i.e., the 'code as law'), and regulation of cross-border payment systems that could include hacking.

\subsubsection{Cryptocurrency market}

This category included studies of the cryptocurrency market, cryptocurrency entrepreneurs, the market's reaction to regulatory actions, and the risks associated with an unregulated cryptocurrency market.

\subsubsection{Cybercrime economy}

This category included studies of the economic impact of blockchain-based micropayment systems and financial inclusion, the use of artificial intelligence to "de-anonymize" the Bitcoin ecosystem, the identification of risk counterparties and potential cybercriminal and illegal trades on the darknet, the system to monitor transactions occurring on the blockchain to detect illegal transactions, and blockchain technology used to prevent double-spending attacks.

\subsubsection{Money laundering}

This category included studies of recent developments regarding anti-money laundering legislation, the analysis of the money laundering process and how the cryptocurrencies have been integrated into this process, and the regulatory and government bodies responding to the regulatory challenges posed by cryptocurrency.

This section provides a summary of the current research topics on cryptocurrency regulation. The results could help other researchers and practitioners gain a better understanding of the current research topics and better conduct future research on this topic.

\subsection{RQ3: What are the research contributions to cryptocurrency regulation?}

This section presents the current research contributions to cryptocurrency regulation. This research question is motivated by the need to understand what is being proposed to resolve the various problems associated with unregulated cryptocurrency through the scanning for research contributions of selected papers.

We classified the research contribution type following the classification of [48] and [55]. We read the abstracts and we identified the type of contribution specified by the author in the abstract. When the abstracts were of too poor quality to ensure the type of contribution, we chose to study the introduction and the conclusion sections of the paper.

We classify the research contribution as follows:

- Framework is a real or conceptual structure intended to serve as a support or guide to cryptocurrency regulation.

- Knowledge is a contribution that is not classified by the author such as tool, protocol, model, method or framework. This type of contribution reveals several aspects of cryptocurrency such as social, financial, legal and technological scope to express the regulatory need. 
Table 3 Research contributions

\begin{tabular}{ll}
\hline Research contribution & Papers \\
\hline Tool & {$[35,56,57]$} \\
Protocol & {$[59,58]$} \\
Model & {$[15,19,38,60]$} \\
Method & {$[18,61]$} \\
Knowledge & {$[4,6,9,11,22,23,31,34,37,64-66]$} \\
Framework & {$[3,5,7,8,20,21,47,62,63]$} \\
\hline
\end{tabular}

- Method is a guideline, procedure, methodology or steps proposed to regulate cryptocurrency.

- Model is a set of contributions for improving the investigation on economic and social dimensions of cryptocurrency regulations.

- Protocol is a technological solution with a set of roles implemented in blockchain technology, proposed to regulate cryptocurrency micropayments and data exchange through a secured channel in distributed ledger platforms.

- Tool is an information system, based on blockchain, proposed to regulate cryptocurrency.

Table 3 shows the identified contributions and their authors.

\subsubsection{Tool}

Three tools were proposed to regulate cryptocurrency regulation. First is an information system to manage cryptocurrency through distributed consensus-based methods [56]. The second is an information system to reduce the aggregated cost of KYC in a jurisdiction using DLT where the main gain is the avoidance of the same tasks being duplicated by different financial institutions [35]. The third tool proposed is a blockchain monitoring system which monitors blocks and transactions generated in Bitcoin and Ethereum networks where the data collected is used to detect illegal transactions in the future [57].

\subsubsection{Protocol}

Two protocols were proposed. One is an application layer payment protocol named Streaming Data Payment Protocol (SDPP) that supports the emerging "Data-for-value" applications where the client-server architecture of SDPP through TCP allows the seller and the buyer to exchange data through a secured channel. The technology-agnostic design of SDPP allows the application developers to easily support different cryptocurrencies and distributed ledger platforms to handle micropayments and the recording of transactions [58].

The second protocol proposed uses the POW (proof-ofwork) technique to allow distributed decision making within a network, namely Bitcoin's blockchain protocol and technology, to give better protection against double-spending attacks [59].

\subsubsection{Model}

We found four proposed models. The first analysed the central bank incentives to establish a network model that includes hacking and proposed a model to calculate an optimal level of central regulation to reduce the critical mass of Bitcoin users, considering the central bank loss function [60].

The second model provides an investigation for understanding the impact of cryptocurrencies on consumer acceptance proposing the contribution of a cryptocurrency acceptance model [15].

The third model investigated factors that influence an individual's intention to use a blockchain cryptocurrency, categorized and identified factors that could influence the intention to use cryptocurrency (specifically to transact) employing a theory of planned behaviour, and proposed a model to understand the acceptance and adoption of cryptocurrency [19].

The fourth is a proposed cryptocurrency accounting model under IFRS to be useful information for users of financial statements when cryptocurrencies are acquired for investment purposes [38].

\subsubsection{Method}

We found two methods. The first method investigated the security issues of blockchain-based transactions on 5G networks, particularly in the mobile sector, and proposed a methodology for policy specification and verification of transactions based on smart contracts in next-generation mobile networks [18].

The second developed and validated a novel method for "de-anonymizing" the Bitcoin blockchain transactions providing the first estimation of different entity types in the Bitcoin blockchain ecosystem and a solution of a prototype implementation for practitioners and regulators which can be used as a tool to assess transactions [61].

\subsubsection{Frameworks}

The nine frameworks found for cryptocurrency regulation were contributions involving different research scopes such as an overview of cryptocurrency [62], an overview on central bank digital currency [20], the challenges posed by distributed governance [63], the financial and monetary policy risks of digital currencies [5], the various risks associated with an unregulated cryptocurrency market [7], the private cryptocurrencies as an integral part of the financial market 
Fig. 5 Research contributions

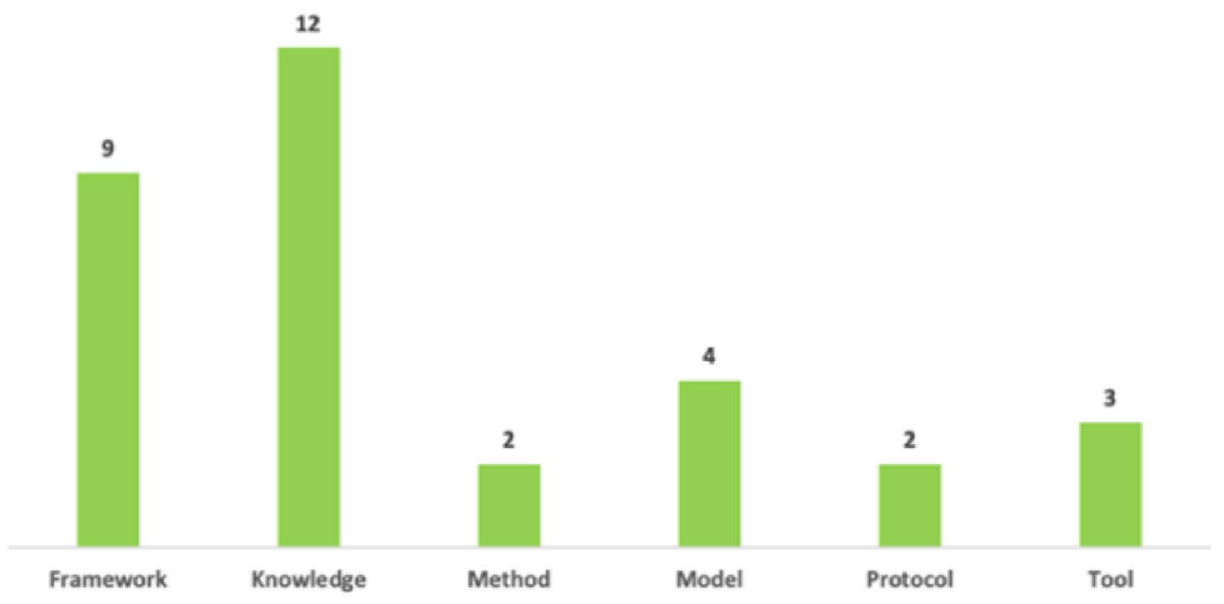

[21], the analyses of virtual currency regulation under financial and economic law, with particular attention to recent developments regarding anti-money laundering legislation [8], and cryptocurrencies from a business ethics perspective [3].

\subsubsection{Knowledge}

The knowledge contributions to the financial sector of cryptocurrency regulation included an overview of financial and monetary policy risks of digital currencies [31], the diverse aspects of virtual currencies and their ability to provide the answer as to whether these new types of currencies could in any way lower the role of nationally emitted money [64], the financial intermediation as a market where the cryptocurrency entrepreneurs will find most difficulty in operating outside existing regulatory regimes [11], and an examination thought-event study on whether and how regulatory actions and communications about such actions have affected cryptocurrency markets [22].

The knowledge contributions interconnecting the financial, legal and technological domain of research on cryptocurrency regulation were: the currencies in the digital sphere, along with their advantages and risks linked with unreasonable usage [65]; the contribution of blockchainbased micropayments to the cybercrime economy [34]; how blockchain might—or might not—be compatible with established legal and regulatory models such as data protection [37]; and the Security and Exchange Commission's initial statements and subsequent pronouncements on Initial Coin Offerings (ICOs) to illustrate the potential problems with applying an older legal framework to an ever-evolving ecosystem [4].

The knowledge contributions interconnecting the legal and technological domain of research on cryptocurrency regulation were: 'regulation by blockchain', examination of the intersection and interactions between conventional law produced and enforced by national legal systems ('code of law') and the internal rules of blockchain systems, which take the form of executable software code operating across a distributed computing network ('code as law') [66]; and Central Bank Digital Currency (CBDC) legal regulation [23].

We also observed knowledge contributions interconnecting problems in various countries related to unregulated cryptocurrency, such as the legal framework of cryptocurrency in various countries [6], the use of cryptocurrencies in the money laundering process, and how regulatory and government bodies are responding to this new form of currency [9].

Figure 5 shows that in terms of research contributions, the current research found 9 frameworks (28.1\%), 12 knowledge (37.5\%), 2 methods (6.3\%), 4 models (12.5\%), 2 protocols (6.3\%), and 3 tools $(9.3 \%)$.

Figure 6 shows the bubble graphs associating research contribution with the research method (left) and research type (right) of the included studies. The vertical axis (research contribution) is shared between the two graphs. The size and shade of each bubble represent the absolute frequency of cryptocurrency regulation papers belonging to a given pair of research contribution and research method (left) or research type (right). The horizontal and vertical axes labels are accompanied by the relative frequency (i.e., percentage) of the class.

The contributions from experiment research method and experience research type were tools and protocols to regulate cryptocurrency.

The model contribution is primarily derived from the evaluation research type and case study research method. The papers that contributed most to the method of cryptocurrency regulation are associated with experiment research method, and solution and validation research types.

We observed that the knowledge contributions come mainly from opinion and philosophical papers using action 


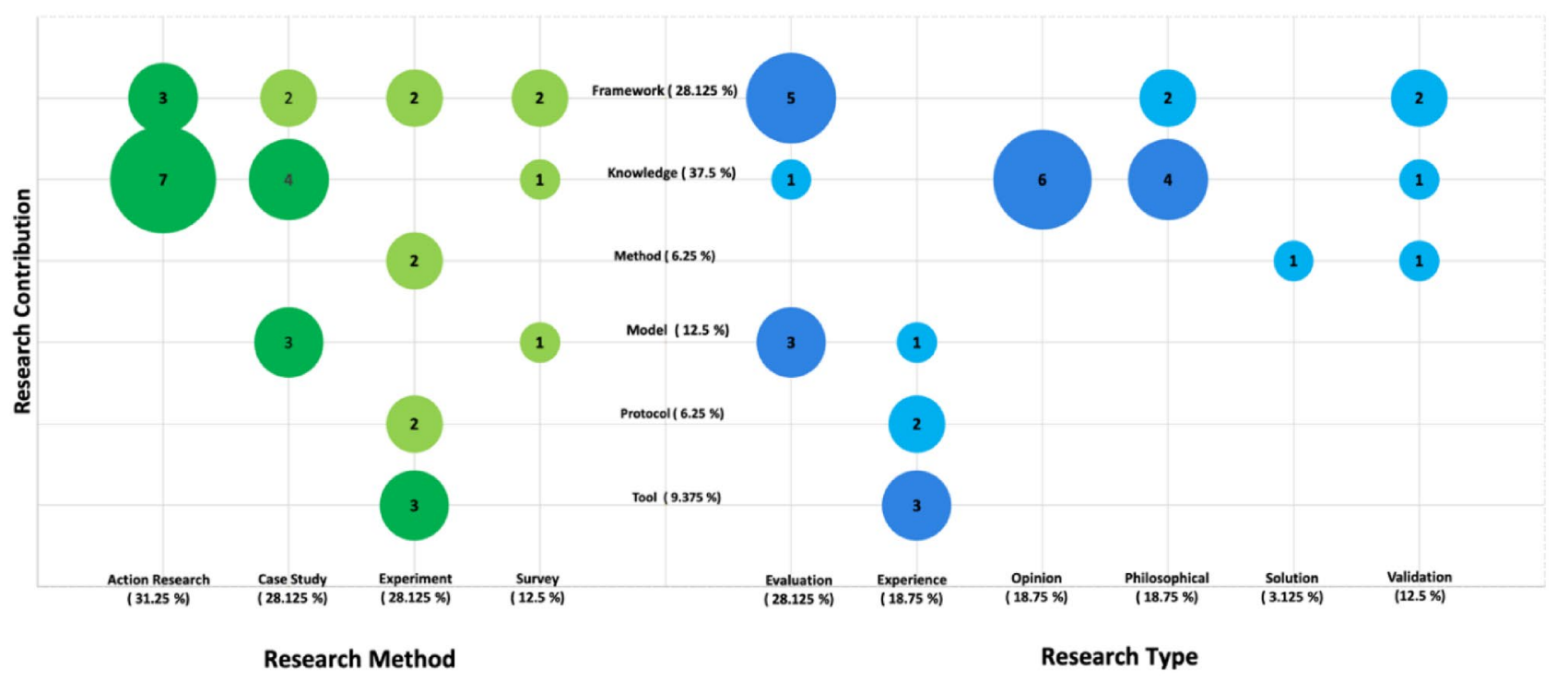

Fig. 6 Visualization of a systematic map in the form of a bubble plot

research and case study research methods. The framework contributions are most associated with evaluation and validation research type studies balanced across all research methods.

This section provides an examination of current research proposals to regulate cryptocurrency, mapping how the research is conducted and identifying the research contributions to cryptocurrency regulatory solutions. These research results could help researchers take the research on cryptocurrency regulation even further.

\subsection{RQ4: what are the research challenges for cryptocurrency regulation?}

This section analyses the research challenges for cryptocurrency regulation. This question is motivated by the need to know the current research challenges for cryptocurrency regulation to help other researchers and practitioners focus their research on the areas that require more attention.

Table 4 shows the identified challenges. We considered the research challenges as a set of problems that need to be solved in future research. In this scope, we identified gaps in the current research as pointed out by authors of selected papers and we listed problems that need to be solved in future work to complement the current research.

\subsubsection{Cryptocurrency regulation}

Calls for future empirical studies on the causes and consequences of financial regulation in blockchain and cryptocurrencies are needed to better understand the appropriate legal and surveillance/enforcement regimes that will minimize negative externalities from blockchain applications, while not stifling innovation [4]. This author also pointed further, to the need for additional work on international cooperation and harmonization of regulation, as financial innovations with blockchain are designed with little regard to national borders [4].

More clarity on the precise legal status of virtual currencies and more regulatory initiative is needed to mitigate the other potential risks of cryptocurrency [8]. Current debates surrounding blockchain demonstrated that lawyers still have a lot to learn from specialists in other disciplines, and vice versa, in this case, collaboration that makes it so rewarding to work in the field of technology law and regulation [37].Card-based payments have no well-defined standard to cater to micropayments with cryptocurrency [34]. One of the most important areas to explore in the compliance and standards domain is how security standards in the financial sector can be applied to blockchainbased financial transactions in 5G networks [18].

Table 4 Research challenges

\begin{tabular}{ll}
\hline Research challenges & Papers \\
\hline Cryptocurrency regulation & {$[4,8,18,34,37]$} \\
Cryptocurrency adoption & {$[3,4,15,20]$} \\
Risk of cryptocurrencies & {$[3,19,47]$} \\
Central bank digital currency regulation & {$[62]$} \\
Accounting for cryptocurrencies & {$[38]$} \\
\hline
\end{tabular}




\subsubsection{Cryptocurrency adoption}

The second gap observed is in cryptocurrency adoption with a call for further research on the economic and ethical effects of the design of altcoin liquidity levels, and on how blockchain technology could be used to promote ethical goals in society, by hitching 'mining' to the creation of social or ecological benefits [3].

The implication of blockchain technologies requires indepth multi-disciplinary attention from the academic and scientific perspective but also at the policy-making level, because, careful steps and risk avoidance should be favoured at the decision-making level for implementing and adopting blockchain in public service, central banks and governmental institutions in general [20]. The importance of investigating the role of the disruptive innovation of cryptocurrencies in the acceptance and trust perceived by users concerning the monetary transactions generated in e-commerce is also pointed out by [15].

\subsubsection{Central bank digital currency regulation}

The third gap observed is in Central Bank Digital Currency regulation. According to [62], the current research is not coherent on the design attributes of a possible CBDC because the design properties, technical implementations as well as legal and societal aspects have not been researched thoroughly.

\subsubsection{Accounting for cryptocurrencies}

The fourth gap identified is the accounting for cryptocurrencies with calls for follow-up studies to investigate the accounting practices of companies using cryptocurrencies, focusing first on companies accepting cryptocurrencies as a means of payment, and then on companies investing into cryptocurrencies. Accounting research should pay attention to the practices of (voluntary) disclosures concerning cryptocurrencies which may bring new insights into the informativeness of financial reporting and the interaction of standards and reporting incentives on accounting (disclosure) quality [38].

\subsubsection{The risk of cryptocurrencies}

The fifth gap relates to the risk of cryptocurrencies with a call for future studies to combine a variety of methods to gain additional insight into the issues of cryptocurrency's vulnerability, risk identification and mitigation, regulation and acceptability [47]. Research is needed which highlights both the risks and potential rewards of altcoins for government actors and a rigorous mapping of this spread would enable business actors, among others, to better understand and offset looming volatility risks and generally to make sound policy decisions in this fast-changing field [3]. Also needed is a model to investigate the antecedents in a variety of contexts employing more advanced statistical modeling techniques such as structural equation modeling (SEM) or partial least squares (PLS) [19].

This section analysed the research challenges for cryptocurrency regulation, and created a comprehensive list of the challenges. The results could help other researchers and practitioners focus their research on the areas that require more attention.

\section{Discussion}

In this section, we discuss the answers to the defined research questions.

This systematic mapping identified the research on cryptocurrency regulatory proposals and current challenges that need to be addressed in future studies, and analysed the publication approaches, the current research topics, the research contributions, and the research challenges for cryptocurrency regulation.

Our purpose was to understand the level of maturity of the current research by analysing the research method and type of the selected studies, to map the current research topics, and to evaluate the current research contribution map in cryptocurrency regulation.

The most significant study type identified was action research, which contributed to the acquisition of new theoretical knowledge, and involved researchers and practitioners acting together on problem diagnosis, intervention, and reflective learning. Evaluation research was also an important type of research, and revealed that the research on cryptocurrency regulation had been focused primarily on the problems of cryptocurrency and proposals for cryptocurrency regulation. This type of research also called for more legislation and better law enforcement.

Current research has been focused on cryptocurrency problems such as money laundering, the dark market, drug trafficking, tax evasion by corrupt firms and criminal organizations, hacking, speculation bubbles, energy consumption, credit, operational and liquidity risks, and has also called for increased legislation and improved law enforcement.

The research topics on cryptocurrency regulation are: distributed governance; central bank digital currency (CBDC); monetary policy; cryptocurrency adoption; security; regulation; cryptocurrency market; cybercrime economy; and money laundering.

Table 5 presents a summary of the most important proposals to regulate cryptocurrency as indicated by the research contribution and research focus of the selected papers. 


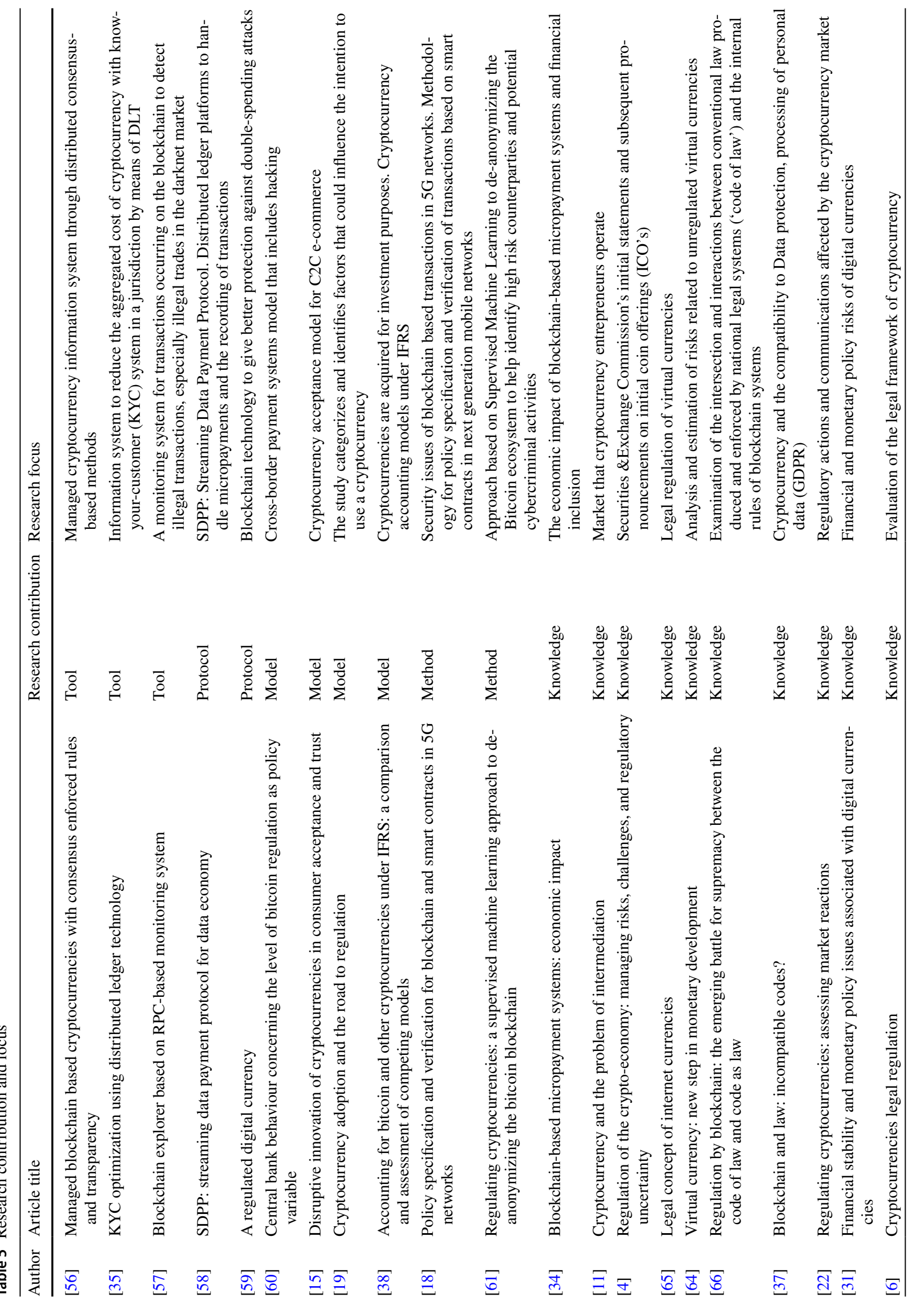




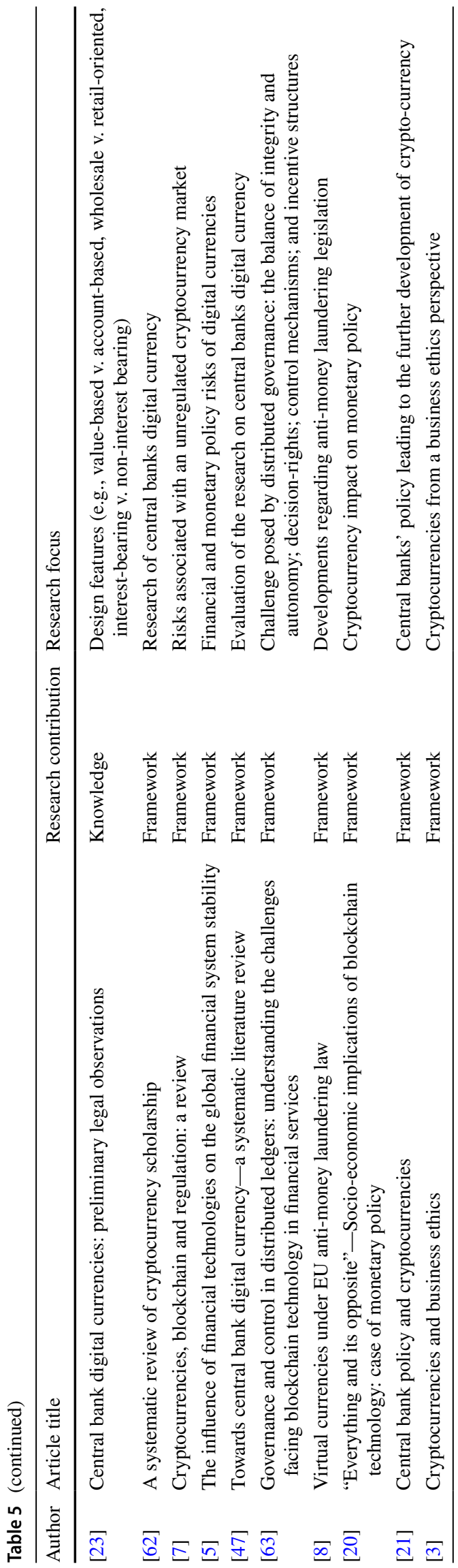

The tools proposed to regulate cryptocurrency regulation included an information system to manage cryptocurrency, and a tool for reducing the aggregated cost of know-yourcustomer (KYC) requirements.

The protocols proposed to regulate cryptocurrency were Streaming Data Payment Protocol (SDPP) and the Bitcoin blockchain protocol which facilitate distributed decision making within a network and give better protection against double-spending attacks.

The models proposed to regulate cryptocurrency were: a model to calculate an optimal level of central regulation; a model to provide an understanding of consumer acceptance of cryptocurrency; and a model to understand the acceptance and adoption of cryptocurrency and cryptocurrency accounting models under IFRS.

The methods proposed to regulate cryptocurrency involved policy specification and verification of transactions based on smart contracts, and a method for de-anonymizing Bitcoin blockchain transactions.

The frameworks proposed for cryptocurrency regulation were Central Bank Digital Currency (CBDC), the challenges of distributed governance, financial and monetary policy risks, and regulation to improve anti-money laundering legislation.

The knowledge contributions of cryptocurrency were: the financial and monetary policy risks of digital currencies; the market for financial intermediation; and the use of blockchain-based micropayments in the cybercrime economy.

More research on the theoretical and conceptual approaches to cryptocurrency regulation is necessary, as well as more projects related to solutions for cryptocurrency regulation. Furthermore, what is also needed are studies focused on the problems of cybercrime and money laundering using cryptocurrency across international borders, which is to say, a global approach to regulating cryptocurrency as an internationally distributed digital currency.

The regulatory research challenges include cryptocurrency regulation, cryptocurrency adoption, Central Bank Digital Currency (CBDC) regulation, accounting for cryptocurrencies and the risks inherent in cryptocurrencies.

Researchers are calling for extended research such as empirical studies on the causes and consequences of financial regulation in blockchain, studies on legal and societal aspects of central bank digital currency, and studies which combine a variety of methods to gain additional insight into the issues of vulnerability, risk identification and mitigation, regulation and acceptability of cryptocurrency.

Cryptocurrency regulation demands multi-disciplinary research to produce a robust regulatory framework including scientific perspective as well as the interests of the key stakeholders such as the regulators and the regulated, especially the International Bank of Settlements (IBS), governments, national and international central banks, banks, anti-money 
laundering organizations, telecommunications regulators and operators, Fintech companies and individuals who use cryptocurrency.

\section{Limitations}

In our study, we identified three limitations that may threaten the validity of the results. We relied on the map of threats to the validity of systematic literature reviews in software engineering [67] to identify limitations and techniques to overcome these limitations.

The first limitation identified was the lack of standard languages with regards to cryptocurrency. Several terminologies such as commodity money, cryptocurrency, cryptographic currencies, digital currency, e-currency, digital cash, electronic currency, e-money, internet-based money, internet currency, virtual currency, virtual money, altcoin, bank digital currency, central bank digital currency were used to introduce the thematic of unregulated cryptocurrency which caused chaos in the research process.

The strategy used to overcome this limitation was to work with another researcher to decide which terms to use in the search strings. We tested this strategy by removing all synonymous terms of cryptocurrency in our search string and observed the results of the search. We adopted this strategy to help eliminate studies that were outside of the scope of the research goal of this review.

The second limitation was the inability to access papers to download, which could have resulted in the absence of relevant primary studies. To resolve this limitation, we did a manual search on Google Scholar and on ResearchGate.

Finally, to overcome the limitation of unsatisfactory data synthesis, which could have influenced the quality of our data analysis, we piloted data synthesis including several different perspectives of cryptocurrency regulation.

\section{Conclusion}

In this systematic mapping study, we identified research that had been conducted on cryptocurrency regulation, as well as current challenges that should be addressed in future studies.

Mapping the publication approaches to cryptocurrency regulation, we concluded that the most significant study type was action research which contributed to the acquisition of new theoretical knowledge, and involved researchers and practitioners acting together on problem diagnosis, intervention, and reflective learning.

The main issues addressed by cryptocurrency research were: solutions for managing cryptocurrency through distributed networks; the design features of cryptocurrency such as value-based, account-based, wholesale, retailoriented, interest-bearing, non-interest bearing; risks of unregulated virtual currencies on monetary policy; and categorization and identification of factors that could influence the intention to use cryptocurrency.

Security of blockchain-based transactions, was another issue addressed by cryptocurrency regulation and covered: the intersection and interactions between conventional law produced and enforced by national legislation; the market reaction in response to regulatory actions; the identification of cybercriminal and illegal trades on the darknet; and the analysis of the money laundering process and how cryptocurrencies have been integrated into this process.

The research contribution on cryptocurrency regulation comprises two types: concrete or tangible proposals such as tools, protocols, methods, models, and, the theoretical or intangible solutions such as frameworks and knowledge.

Researchers are calling for more research via empirical studies related to: the causes and consequences of financial regulation in blockchain; the legal and societal aspects of central bank digital currency; and combinations of various methods to gain additional insight into the vulnerability, risk identification and mitigation, regulation and acceptability of cryptocurrency.

The cryptocurrency regulation research challenges include calls for empirical studies addressing: the causes and consequences of financial regulation in blockchain and cryptocurrencies; the legal and societal aspects of central bank digital currency; international cooperation and harmonization of regulation; regulation to mitigate the potential risks of cryptocurrency; standards for card-based payments and micropayments with cryptocurrency; security standards in the financial sector applied to blockchainbased financial transactions in 5G networks; and standards for cryptocurrencies accounting.

This systematic mapping study contributes to the structuring of research into cryptocurrency regulation, and summarizes the research approaches, topics, contributions and challenges. The results of this systematic mapping could help researchers and practitioners to better understand how research is being done and communicated. This can thus help take the research on cryptocurrency regulation even further, can increase the current research topics, and can increase the research contributions so that researchers and practitioners can better conduct their future research on these topics and more effectively focus on the areas that require more attention.

For future work, we propose to further investigate the stakeholders, the drivers, the assessments, the goals for regulation of cryptocurrency, the central bank digital currency projects and decentralized finance (DeFi). 
Acknowledgements We would like to express our gratitude to the research center INOV INESC Inovação. We would also like to thank Garth Bernard for the paper revision.

Author contributions MMS coordinated the study, participated in the design of the research protocol, oriented the statistical analysis of research results, and helped in drafting the manuscript. ECS carried out the theoretical background of blockchain-based cryptocurrency regulation, conceived the study, performed the statistical analysis, and wrote the manuscript's draft. All authors read and approved the final manuscript.

Funding Research reported in this publication was supported by Foundation for Science and Technology (FCT) - Portugal through the individual research grant SFRH/BD/151432/2021.

Availability of data and material Not applicable.

Code availability Not applicable.

\section{Declarations}

Conflict of interest The authors declare that they have no competing interests.

Ethics approval Not applicable.

Consent to participate Not applicable.

Consent for publication Not applicable.

\section{References}

1. Crosby M, Nachiappan PP, Verma S, Kalyanaraman V (2016) BlockChain technology: beyond bitcoin. Appl Innov Rev 2:71

2. Nakamoto S. (2008) 'Bitcoin: a peer-to-peer electronic cash system', [Online]. Available: https://bitcoin.org/bitcoin.pdf. Accessed $07 \mathrm{Feb} 2019$

3. Dierksmeier C, Seele P (2018) Cryptocurrencies and business ethics. J Bus Ethics 152(1):1-14. https://doi.org/10.1007/ s10551-016-3298-0

4. Cumming DJ, Johan S, Pant A (2019) Regulation of the cryptoeconomy: managing risks, challenges, and regulatory uncertainty. J Risk Financ Manag 12(3):126. https://doi.org/10.3390/jrfm1 2030126

5. Azarenkova G, Shkodina I, Samorodov B, Babenko M, Onishchenko I (2018) The influence of financial technologies on the global financial system stability. Invest Manag Financ Innov 15(4):229-238. https://doi.org/10.21511/imfi.15(4).2018.19

6. Cvetkova I (2018) Cryptocurrencies legal regulation. BRICS Law J 5(2):128-153. https://doi.org/10.21684/ 2412-2343-2018-5-2-128-153

7. Afzal A (2019) Cryptocurrencies, blockchain and regulation: a review. Lahore J Econ 24(1):103-130. https://doi.org/10.35536/ lje.2019.v24.i1.a5

8. Vandezande N (2017) Virtual currencies under EU anti-money laundering law. Comput Law Secur Rev 33(3):341-353. https:// doi.org/10.1016/j.clsr.2017.03.011

9. Albrecht C, Duffin KM, Hawkins S, Rocha VM (2019) The use of cryptocurrencies in the money laundering process. J Money Laund Control. https://doi.org/10.1108/JMLC-12-2017-0074
10. Auer R, Claessens S (2020) Cryptocurrency market reactions to regulatory news. CEPR Discussion Paper. https://ssrn.com/abstr act $=3594224$. Accessed 19 Aug 2020

11. Harwick C (2016) Cryptocurrency and the problem of intermediation. Indep Rev 20(4):569-588

12. B. of England (2020) Central bank digital currency opportunities, challenges and design. Discussion paper. https://www.banko fengland.co.uk/paper/2020/central-bank-digital-currency-oppor tunities-challenges-and-design-discussion-paper. Accessed 1 July 2020

13. Petersen K, Vakkalanka S, Kuzniarz L (2015) Guidelines for conducting systematic mapping studies in software engineering: an update. Inf Softw Technol 64:1-18. https://doi.org/10.1016/j. infsof.2015.03.007

14. Lee I, Shin YJ (2018) Fintech: ecosystem, business models, investment decisions, and challenges. Bus Horiz 61(1):35-46. https:// doi.org/10.1016/j.bushor.2017.09.003

15. Mendoza-Tello JC, Mora H, Pujol-López FA, Lytras MD (2019) Disruptive innovation of cryptocurrencies in consumer acceptance and trust. Inf Syst E-bus Manag 17(2-4):195-222. https://doi.org/ 10.1007/s10257-019-00415-w

16. Patric Schueffe N, Groeneweg, and R. Baldegger, (2019) The Crypto Enciclopedia - Coins. Growth Publisher, Bern, Tokens and digital Assets from A to Z, p 1386

17. Akhtar Z (2019) 'From blockchain to hashgraph: distributed ledger technologies in the wild', in proceedings-2019 international conference on electronics and computer engineering. UPCON. https://doi.org/10.1109/UPCON47278.2019.8980029

18. Unal D, Hammoudeh M, Kiraz MS (2019) Policy specification and verification for blockchain and smart contracts in $5 \mathrm{G}$ networks. ICT Express 6(1):43-47. https://doi.org/10.1016/j.icte. 2019.07.002

19. Schaupp LC, Festa M (2018) Cryptocurrency adoption and the road to regulation. Annu Int Conf Digit Gov Res. https://doi.org/ $10.1145 / 3209281.320$

20. Ben Dhaou SI, Rohman IK (2018) 'Everything and its oppositesocio-economic implications of blockchain technology: case of monetary policy', ICEGOV'18 'everything and its opposite. Proc 11th Int Conf Theory Pract Electron Gov. https://doi.org/10.1145/ 3209415.3209502

21. Krivoruchko S, Ponamorenko V, Nebera A (2018) Central bank policy and cryptocurrencies. J Rev Global Econ 7:549-561

22. Auer R, Claessens S (2018) Regulating cryptocurrencies: assessing market reactions. BIS Q Rev. https://ssrn.com/abstract=32880 97. Accessed 9 Mar 2020

23. Nabilou H (2019) Central bank digital currencies: preliminary legal observations. J Bank Regul. https://doi.org/10.2139/ssrn. 3329993

24. Boar C, Holden H, Wadsworth A (2020) Impending arrival - a sequel to the survey on central bank digital currency. Ssrn. https:// www.bis.org/publ/bppdf/bispap107.pdf. Accessed 27 Aug 2020

25. Boar C, Wehrli A (2021) Ready, steady, go?-Results of the third BIS survey on central bank digital currency, no. 114

26. Dantes D (2021) Bank of America Sees DeFi "Potentially More Disruptive Than Bitcoin"', CoinDesk, [Online]. Available: https:// www.coindesk.com/bank-of-america-defi-potentially-more-disru ptive-than-bitcoin. Accessed 15 Jul 2021

27. B. of America (2021) 'Commodity strategist—bitcoin's dirty little secret', 2021 [Online]. Available: https://rsch.baml.com/access? q=JLQz8CGIkJs. Accessed 15 Jul 2021

28. Napoletano E, Schmidt J (2021) Decentralized finance is building a new financial system, 2021 [Online]. Available: https:// www.forbes.com/advisor/investing/defi-decentralized-finance/. Accessed 15 Jul 2021

29. Emmanuel OO (2021) 'Citibank demystifies MakerDAO and DeFi for fund managers I BTCMANAGER', https://btcmanager.com/, 
16-Apr-2021 [Online]. Available: https://btcmanager.com/citib ank-makerdao-defi-fund-managers/. Accessed 14 Jul 2021

30. Maker DAO (2021) 'MakerDAO documentation - Maker Docs', 2021. [Online]. Available: https://docs.makerdao.com/. Accessed 15 Jul 2021

31. Nelson B (2018) Financial stability and monetary policy issues associated with digital currencies. J Econ Bus. https://doi.org/10. 1016/j.jeconbus.2018.06.002

32. S. of Essays, 'Regulation in the Era of Fintech I The Regulatory Review', The Regulatory Review, 2021 [Online]. Available: https://www.theregreview.org/2021/04/26/regulation-in-era-finte $\mathrm{ch} /$. Accessed $15 \mathrm{Jul} 2021$

33. Mitra S, Karathanasopoulos A (2020) FinTech revolution: the impact of management information systems upon relative firm value and risk. J Bank Financ Technol 4:175-187. https://doi.org/ 10.1007/S42786-020-00023-0

34. Khan N, Ahmad T, Staete R (2019) Blockchain-based micropayment systems: economic impact, IDEAS '19. Proc 23rd Int Database Appl Eng Symp Database Appl Eng Symp. https://doi.org/ 10.1145/3331076.3331096

35. Larrosa FH, Maune JF, Erazzú LE, Camadro EL (2017) KYC optimization using distributed ledger technology. Bus Inf Syst Eng. https://doi.org/10.1007/s12599-017-0504-2

36. Johnson KN (2021) Decentralized finance: regulating cryptocurrency exchanges. William Mary Law Rev 62:4-5. https://doi.org/ $10.2139 /$ ssrn.3831439

37. Millard C (2018) Blockchain and law: incompatible codes? Comput Law Secur Rev 34(4):843-846. https://doi.org/10.1016/j.clsr. 2018.06.006

38. Procházka D (2018) Accounting for bitcoin and other cryptocurrencies under IFRS: a comparison and assessment of competing models. Int J Digit Account Res 18:161-188. https://doi.org/10. 4192/1577-8517-v18_7

39. BIS, 'BIS Innovation Hub work on central bank digital currency (CBDC)', Bank for International Settlements (BIS), 2021. [Online]. Available: https://www.bis.org/about/bisih/topics/cbdc. htm?m=1\%7C441\%7C714\%7C98. Accessed 17 Jul 2021

40. Bouilhet A (2021) Eurosystem launches digital euro project', European Central Bank (ECB), 2021. [Online]. Available: https:// www.ecb.europa.eu/press/pr/date/2021/html/ecb.pr210714 d9919 8ea23.en.html. Accessed 17 Jul 2021

41. R. Auer, G. Cornelli, and J. Frost, 'Rise of the central bank digital currencies: drivers, approaches and technologies', 2021 [Online]. Available: https://www.bis.org/publ/work880.htm. Accessed 17 Jul 2021

42. Wilson T, Jones H (2021) Binance, the giant crypto exchange under regulatory scrutiny | Reuters, Reuters, 01-Jul-2021 [Online]. Available: https://www.reuters.com/world/china/binan ce-giant-crypto-exchange-under-regulatory-scrutiny-2021-07-01/. Accessed 17 Jul 2021

43. B. Teams (2021) Ceasing Support for Stock Tokens on Binance. com and Migration to CM-Equity AG for EEA Users I Binance Support, Binance, 2021. [Online]. Available: https://www.binan ce.com/en/support/announcement/3a0304f3ee1c43668959c1b01 f610d59. Accessed 18 Jul 2021

44. Sandner P (2021) Decentralized finance will change your understanding of financial systems, Forbes, 2021 [Online]. Available: https://www.forbes.com/sites/philippsandner/2021/02/22/decen tralized-finance-will-change-your-understanding-of-financialsystems/?sh=5d9b4eb45b52. Accessed 15 Jul 2021

45. McLaughlin T (2021) A treasurer's guide to the future of money, Citi Bank, 2021. [Online]. Available: https://www.citibank.com/ tts/insights/articles/article61.html. Accessed 18 Jul 2021

46. Kitchenham B, Budgen D, Brereton OP (2010) The value of mapping studies-a participant-observer case study. Int Conf Eval Assess Softw Eng. https://doi.org/10.14236/ewic/ease2010.4
47. Tronnier F, Recker M, Hamm P (2020) Blockchain application for central banks : a systematic mapping study', in twenty-third pacific asia conference on information systems, Dubai, UAE, 2020 [Online]. Available: https://aisel.aisnet.org/pacis2020/131/

48. Dashkevich N, Counsell S, Destefanis G (2020) Blockchain application for central banks: a systematic mapping study. IEEE Access. Available: https://ieeexplore.ieee.org/document/9149861

49. Petersen K, Feldt R, Mujtaba S, Mattsson M (2008) Systematic mapping studies in software engineering. Int Conf Eval Assess Softw Eng EASE. https://doi.org/10.14236/EWIC/EASE2008.8

50. Kitchenham B, Charters S (2007) Guidelines for performing systematic literature reviews in software engineering [Online]. Available: http://citeseerx.ist.psu.edu/viewdoc/summary?doi=10.1.1. 117.471. Accessed 26 Nov 2019

51. Liberati A et al (2009) The PRISMA statement for reporting systematic reviews and meta-analyses of studies that evaluate health care interventions: explanation and elaboration. J Clin Epidemiol 151(4):e1-e34. https://doi.org/10.1016/j.jclinepi.2009.06.006

52. Petersen K, Bin Ali N (2011) Identifying strategies for study selection in systematic reviews and maps. Int Symp Empir Softw Eng Meas. https://doi.org/10.1109/esem.2011.46

53. Sjøberg DIK, Dybå T, Jørgensen M (2007) The future of empirical methods in software engineering research. FoSE Futur Softw Eng. https://doi.org/10.1109/FOSE.2007.30

54. Wieringa R, Maiden N, Mead N, Rolland C (2006) Requirements engineering paper classification and evaluation criteria: a proposal and a discussion. Requir Eng 11(1):102-107. https://doi.org/10. 1007/s00766-005-0021-6

55. Karampela M et al (2019) The extent and coverage of current knowledge of connected health: systematic mapping study. J Med Internet Res. https://doi.org/10.2196/14394

56. Mell P (2018) Managed blockchain based cryptocurrencies with consensus enforced rules and transparency, in 2018 17th IEEE international conference on trust, security and privacy in computing and communications/12th IEEE international conference on big data science and engineering (TrustCom/BigDataSE). IEEE. https://doi.org/10.1109/TrustCom/BigDataSE.2018.00177

57. Lee C, Kim H, Maharjan S, Ko K, Hong JWK (2019) Blockchain explorer based on RPC-based monitoring system, ICBC 2019. IEEE Int Conf Blockchain Cryptocurr. https://doi.org/10.1109/ BLOC.2019.8751468

58. Radhakrishnan R, Ramachandran GS, Krishnamachari B (2019) SDPP: streaming data payment protocol for data economy, ICBC 2019. IEEE Int Conf Blockchain Cryptocurr. https://doi.org/10. 1109/BLOC.2019.8751291

59. Wu Y, Fan H, Wang X, Zou G (2019) A regulated digital currency. Sci China Inf Sci 62(3):32109. https://doi.org/10.1007/ s11432-018-9611-3

60. Sauer B (2015) Central bank behaviour concerning the level of bitcoin regulation as a policy variable. Athens J Bus Econ 1(4):273286. https://doi.org/10.30958/ajbe.1-4-1

61. Yin HHS, Langenheldt K, Harlev M, Mukkamala RR, Vatrapu R (2019) Regulating cryptocurrencies: a supervised machine learning approach to de-anonymizing the bitcoin blockchain. J Manag Inf Syst 36(1):37-73. https://doi.org/10.1080/07421222.2018. 1550550

62. Adeleke I, Zubairu UM, Abubakar B, Maitala F, Mustapha Y, Ediuku E (2019) A systematic review of cryptocurrency scholarship. Int J Comm Financ 5(2):63-75

63. Zachariadis M, Hileman G, Scott SV (2019) Governance and control in distributed ledgers: understanding the challenges facing blockchain technology in financial services. Inf Organ 29(2):105117. https://doi.org/10.1016/j.infoandorg.2019.03.001

64. Dibrova A (2016) Virtual currency: new step in monetary development. Procedia Soc Behav Sci 229:42-49. https://doi.org/10. 1016/j.sbspro.2016.07.112 
65. Kisiel D (2018) Legal concept of internet currencies. Financ Law Rev 11(3):1. https://doi.org/10.4467/22996834FLR.18.013.9215

66. Yeung K (2019) Regulation by blockchain: the emerging battle for supremacy between the code of law and code as law. Mod Law Rev. https://doi.org/10.1111/1468-2230.12399
67. Zhou X, Jin Y, Zhang H, Li S, Huang X (2016) A map of threats to validity of systematic literature reviews in software engineering. Proc Asia-Pacific Softw Eng Conf APSEC. https://doi.org/ 10.1109/APSEC.2016.031 